\title{
Ultrastructure and Cytochemistry of the Tegument of Atriaster heterodus (Platyhelminthes: Monogenea)
}

\author{
Cláudia Portes Santos $/{ }^{+}$, Reinalda Marisa Lanfredi*
}

Universidade Santa Úrsula, Instituto de Ciências Biológicas e Ambientais, Rua Fernando Ferrari 75, 22231-040 Rio de Janeiro, RJ, Brasil *Laboratório de Helmintologia, Instituto de Biofísica Carlos Chagas Filho,

Universidade Federal do Rio de Janeiro, Rio de Janeiro, RJ, Brasil

The tegument of the polyopisthocotylean monogenean Atriaster heterodus Lebedev \& Parukhin, 1969 was studied using transmission electron microscopy. The outer syncytial layer of the tegument is connected to the internal cell bodies by cytoplasmic extensions which interweave between the muscular fibres. The free surface of the syncytium has projections of the external membrane which are similar to microvilli. The undulating basal membrane, with numerous narrow elongate projections, is associated with the basal lamina situated between the syncytial and muscular layers. The cell bodies and syncytial layer of the tegument exhibit two types of vesicles, one with fibrous contents and one with electrondense contents; these were analysed using two cytochemical tests, the E-PTA and alcian blue methods, used for the first time on monogeneans.

Key words: Atriaster heterodus - tegument - ultrastructure - cytochemistry

The morphology and topography of Atriaster heterodus Lebedev \& Parukhin, 1969 have been previously studied by Euzet and Maillard (1973), Mamaev (1984) and Santos et al. (1996), and the ultrastructure of gastrodermis by Santos et al. (1998). The ultrastructure of the outer layer of the tegument of Atriaster sp. collected from the fish Diplodus sargus off the coast of Senegal, has been briefly studied by Justine (1992), who considered it close to A. heterodus. The observations presented here show for the first time the ultrastructure of the whole tegument of a species of the genus from the coast of Brazil, as well as the nature of some cytoplasmic vesicles within the tegument, examined using histological and cytochemical tests.

\section{MATERIALS AND METHODS}

Parasites - Specimens of A. heterodus were obtained from the gills of $D$. argenteus (Val., 1830) (Sparidae) collected off Copacabana beach, Rio de Janeiro, Brazil.

Histology - Monogeneans were fixed in 5\% buffered formalin or $70 \%$ alcohol. Specimens were embedded in paraffin wax and sectioned at $5 \mu \mathrm{m}$

Financial support by CNPq, Conselho Nacional de Pesquisa, and Pronex, Programa de Núcleos de Excelência

${ }^{+}$Corresponding author. Fax: +55-21-551.6446. E-mail: cpsantos@ax.apc.org

Received 2 February 2000

Accepted 6 June 2000 and stained in toluidine blue following Pearse (1968) or alcian blue-PAS after McManus and Mowry (1960). Stained sections were cleared in xylene and mounted in balsam.

Ultrastructure - Parasites were fixed for $2 \mathrm{~h}$ at $4{ }^{\circ} \mathrm{C}$ in a solution containing $4 \%$ paraformaldehyde, $2.5 \%$ glutaraldehyde and sucrose $0.3 \mathrm{M}$ in $0.1 \mathrm{M}$ cacodylate buffer at $\mathrm{pH} 7.2$ diluted 1:1 in sea water. After washing in the same solution, they were post-fixed for $1 \mathrm{~h}$ in a solution containing $1 \% \mathrm{OsO}_{4}$ in $0.1 \mathrm{M}$ cacodylate buffer, dehydrated in acetone and embedded in Epon. Ultrathin sections were picked up on uncoated 200 mesh copper grids, double-stained with uranyl acetate and lead citrate, and observed using Jeol JEM 100CX or Zeiss 900 electron microscopes.

Cytochemistry - For the detection of basic proteins, parasites were fixed as described above for ultrastructure, dehydrated in ethanol and incubated in a solution containing $2 \%$ phosphotungstic acid in absolute ethanol (E-PTA) for $2 \mathrm{~h}$ at room temperature (Bloom \& Aghajanian 1968). Specimens were washed in ethanol, incubated for $10 \mathrm{~min}$ in propylene oxide, and embedded in Epon to be examined without counterstaining. To test for mucopolysacharides, parasites were fixed for $18 \mathrm{~h}$ at room temperature in a solution containing $4 \%$ glutaraldehyde and $1 \%$ alcian blue in sea-water (Benhnke \& Zelander 1970). Samples were washed twice for $10 \mathrm{~min}$ in sea-water and post-fixed for 2 $\mathrm{h}$ at room temperature in dark conditions in a solution containing $1 \% \mathrm{OsO}_{4}, 0.8 \%$ potassium ferricyanide and $5 \mathrm{mM} \mathrm{CaCl}_{2}$ in $0.1 \mathrm{M}$ cacodylate buffer $+3 \%$ sucrose. They were then dehydrated in ac- 
etone and embedded in Epon. Ultrathin sections were picked up on uncoated 200 mesh copper grids, stained with uranyl acetate and lead citrate and examined using a Zeiss 900 or a Jeol JEM 100CX electron microscope.

\section{RESULTS}

The tegument is composed of inner nucleated cell bodies with cytoplasmic connections which interweave between the muscular fibres to connect with the external syncytial layer (Fig. 1). The composition and width of the tegument varies according to its position along the body.

The inner cell bodies have an irregular shape; their large nuclei are sometimes elongate and heterochromatin is well differentiated. In the cytoplasm, clusters of free ribosomes, rough endoplasmic reticulum, mitochondria and various vesicles are observed (Figs 1,2). The cytoplasm, which is sometimes reduced close to the nucleus, extends by cytoplasmic connections throughout the muscular zone, to reach the external syncytium (Figs 3,9 ).

The muscular layer varies in thickness along the body and the longitudinal, oblique and circular fibres are observed (Fig. 1), and are always close to numerous mitochondria (Fig. 9). In the opisthohaptor, the muscular layers between the clamps are well developed, but, in the area immediately adjacent to the clamps, the muscles are reduced with a corresponding increase in the number of cytoplasmic extensions. For the rest of the body, the muscular layer is well organized, its outer layer being composed of circular fibres. Above them there is a basal lamina of uniform width, itself covered by a basal plasma membrane which presents numerous narrow elongate projections which extend into the syncytium (Figs 3, 4). The basal lamina and basal membrane, which undulate, following the contour of the adjacent circular muscle fibres, delimit and support the external tegument (Figs 1, 3).

The surface of the tegument is covered by an external plasma membrane which bears irregular evaginations similar to microvilli. Along the body the number of evaginations is reduced, but on the opisthohaptor, they are found in larger numbers and may be subject to multiple ramification (Figs $1,3)$. The syncytium, delimited by the external and basal membranes, varies in width according to the number of vesicles present within it. Clusters of free ribosomes and arrays of concentric membrane myelin-like figures occur in this region (Fig. 5).

Numerous vesicles are found in the subtegumentary cells as well as in their cytoplasmic extensions (Figs 1,2). They are also found in the syn- cytium and among the ramifications of the external membrane, communicating with the environment (Figs 3-6). The vesicles vary in size and shape, some exhibiting fibrous contents, with or without electron-dense granules, and some are totally electron-dense and in the form of small or large (elongate or spherical) bodies. The electron-dense elongate bodies are always concentrated inside or close to the narrow projections of the basal membrane (Fig. 6).

The presence of a glycocalyx covering the surface plasma membrane was suggested by positive results with the alcian blue-PAS test and for $\beta$ metachromasy, indicated by toluidine blue. In the alcian blue cytochemical test, only the electrondense vesicles indicated the presence of mucopolysacharides, both in the syncytial region and in the cytoplasmic extensions of the subtegumentary cells, while the vesicles with fibrous contents were negative (Fig. 8). The electron-dense vesicles were positive for basic proteins, which also indicated the muscular fibres, mitochondria and nuclear chromatin (Figs 9,10).

\section{DISCUSSION}

The ultrastructure of the tegumental system of A. heterodus demonstrates that despite the presence of only two types of vesicles instead of three previously described in this species, the tegument is compatible with that described for other Platyhelminthes.

Justine (1992) described only the outer layer of the tegument of Atriaster sp. collected from the Senegal coast, referring to an undulating external membrane with some microvilli covering a terminal web and three types of vesicles in the syncytium: spherical with fibrous electron-dense contents; spherical with homogeneous moderately electron-dense contents; and elongate bodies with electron-opaque homogeneous contents. An undulating basal membrane associated with the basal lamina and a fibrous matrix involving muscular cells was also referred to. Neither the internal part of the tegument with its cell bodies and cytoplasmic extensions nor any cytochemical tests had previously been described for this species. In view of this, E-PTA and alcian blue tests were performed and information concerning the tegumental ultrastructure has now been elucidated.

Evaginations of the external membrane similar to those described above for $A$. heterodus, were also found in Atriaster sp. (Justine 1992). However, according to the microvillar pattern (Bretscher 1991) and, in view of the fact that no parallel microfilament bands in the centre of each projection were observed, the term "evagination" rather than "microvillus" was employed here. Their size and 


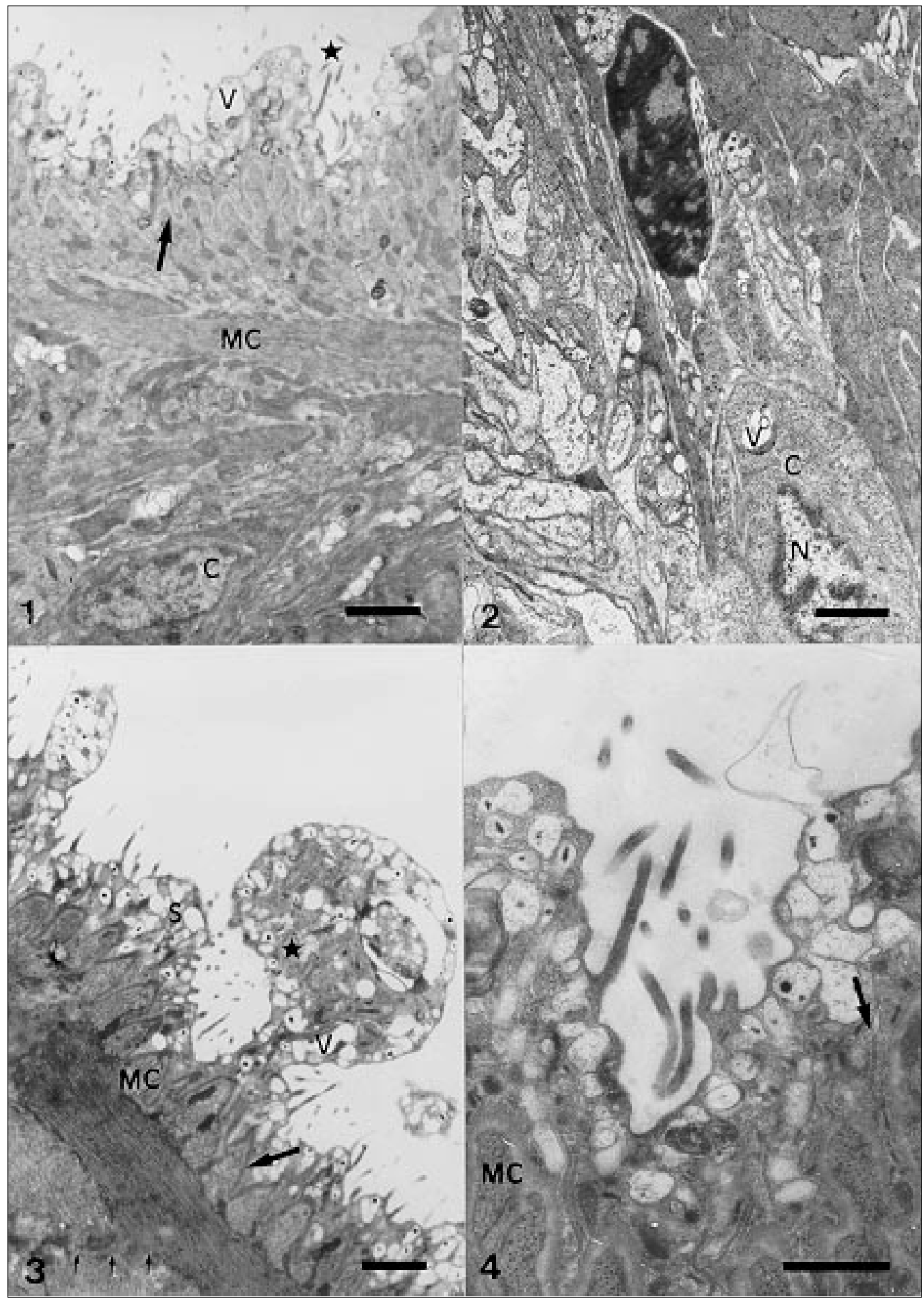

Tegument of Atriaster heterodus. Fig. 1: general view with cell bodies (C) in the inner region and muscular layer (MC), undulating basal lamina (arrow), vesicles (V) and external membranous evaginations (star). Fig. 2: tegumentary cell body with large nucleus $(\mathrm{N})$ and cytoplasm $(\mathrm{C})$ with clusters of free ribosomes and vesicles (V). Fig. 3: tangled evaginations of the syncytium (star) with numerous vesicles (V); muscular layer (MC) is separated from the syncytium (S) by the basal lamina and basal membrane (large arrow); cytoplasmic connection from internal tegument (small arrows). Fig. 4: circular muscle fibres (MC) follow the undulations of the basal lamina and the basal membrane; the latter forms narrow projections which extend into the syncytial region of the tegument (arrow). Bars - Figs 1-3 = $2 \mu \mathrm{m}$; Fig. $4=1 \mu \mathrm{m}$ 


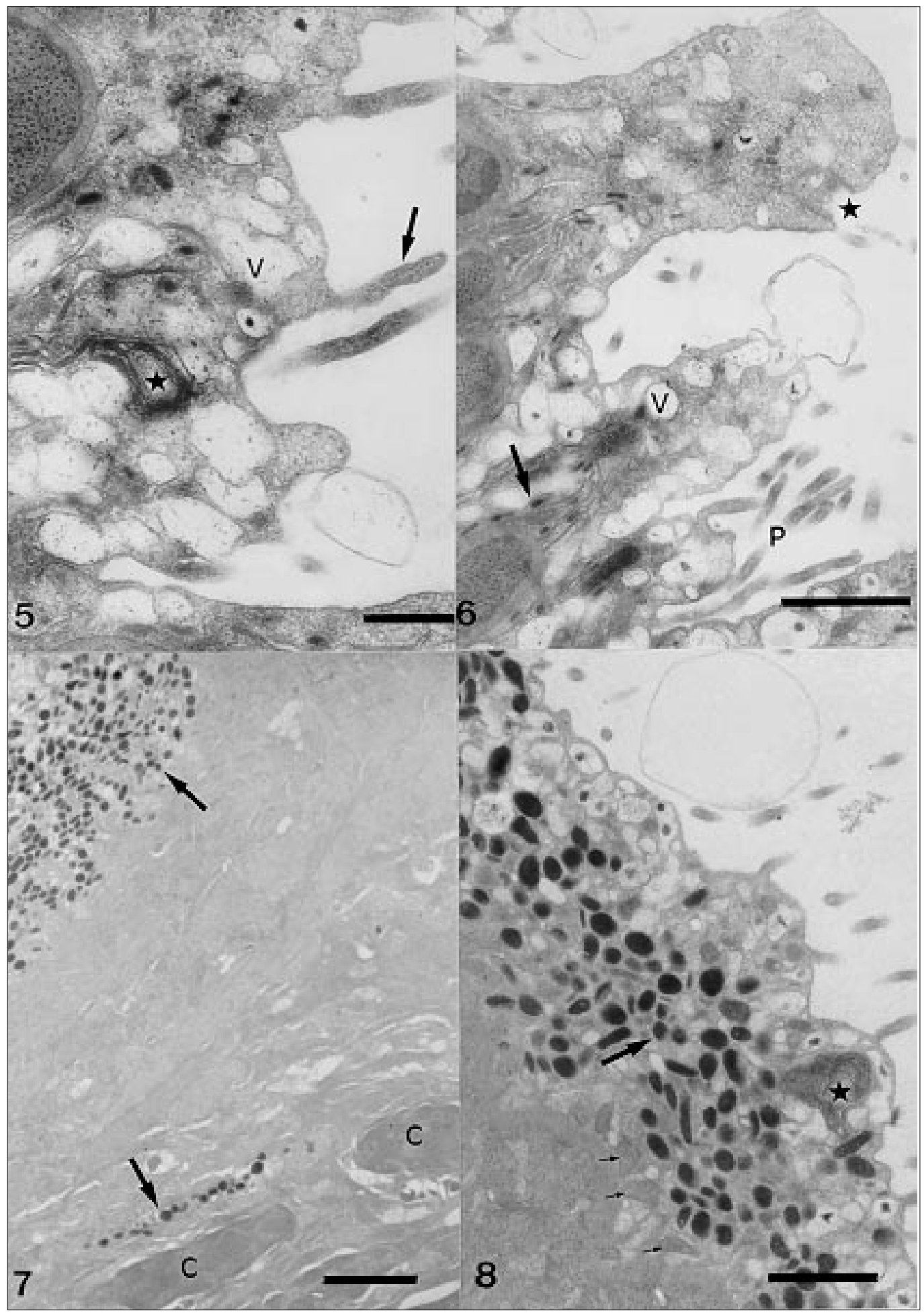

Fig. 5: syncytium with evaginations of the surface membrane (arrow), vesicles (V) and membrane arrays looking like myelin figures (star). Fig. 6: external tegument with evaginations of the membrane (P) and vesicles with fibrous contents (V); elongate electron-dense vesicles (arrow) occur inside and close to the projections of the basal membrane; vesicles open to with the outside (star). Fig. 7: alcian blue positive vesicles (arrows) in the external tegument and in the tegument cell bodies (C). Fig. 8: detail of vesicles (large arrow) and membranous concentric arrays (star) highlighted by the alcian blue method and mitochondria (small arrows). Bars - Fig. $5=0.5 \mu \mathrm{m}$; Figs 6 and $8=1 \mu \mathrm{m}$; Fig. $7=2 \mu \mathrm{m}$ 


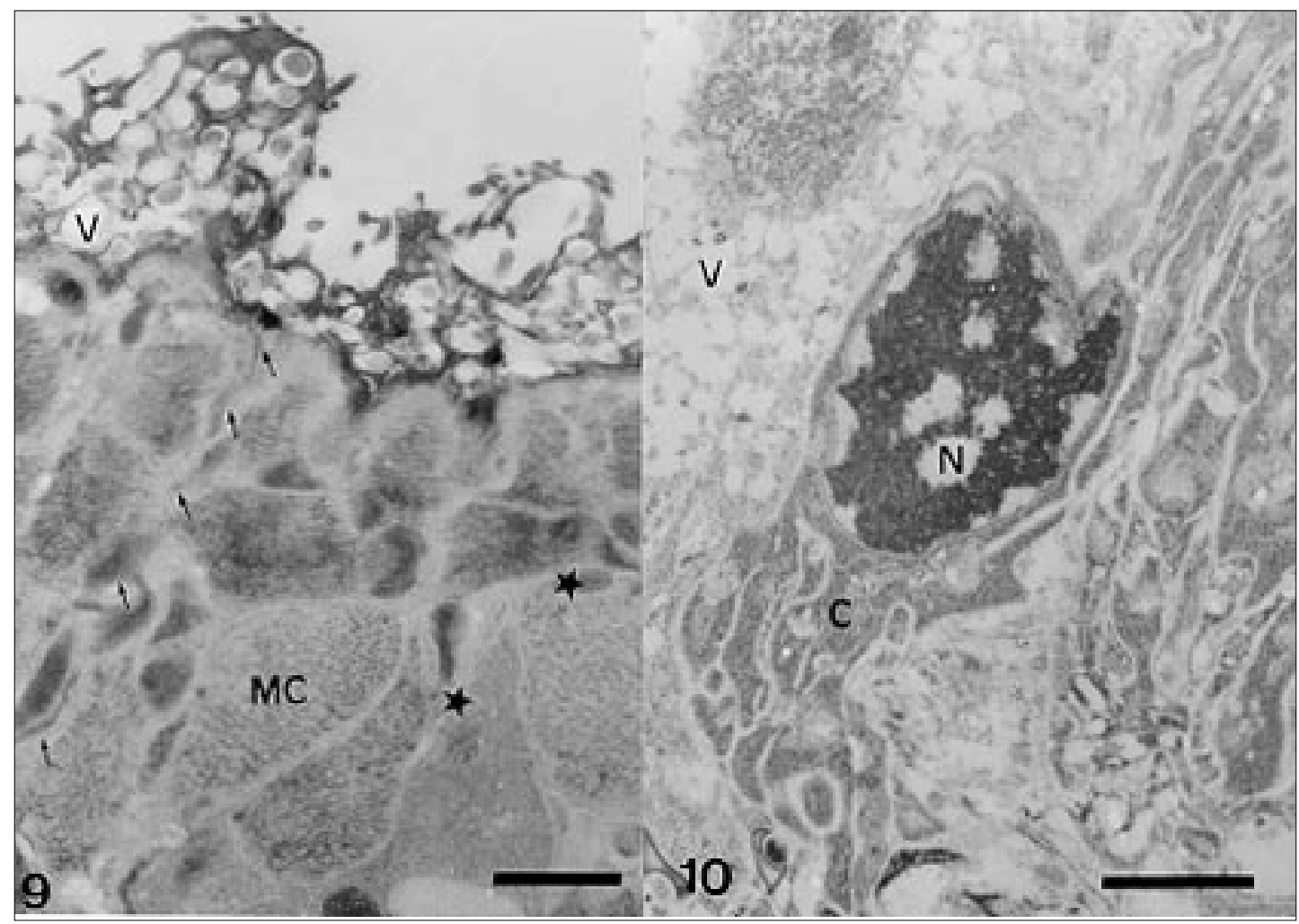

Tegument of Atriaster heterodus stained using the E-PTA method. Fig. 9: structures visible include muscle fibres (MC), mitochondria (star), vesicles (V) and cytoplasmic connection throughout the muscular zone to external syncytium (arrows). Fig. 10: tegumentary cell with electron-dense nucleus $(\mathrm{N})$, cytoplasmic extensions $(\mathrm{C})$ and vesicles $(\mathrm{V})$. Bars - Fig. $9=1 \mu \mathrm{m}$; Fig. $10=$ $2 \mu \mathrm{m}$

distribution along the body was variable, being short and scattered along the body proper and in greater density on the opisthohaptor. A similar pattern in relation to size and distribution of these tegumentary evaginations was referred to by Lyons (1973), who considered that the presence of microvilli in attachment areas could help spread and mix the sticky secretions of different gland-cells, giving protection in a mechanical, osmoregulatory or antibacterial sense. The presence of a glycocalyx covering the surface plasma membrane is suggested by positive alcian blue-PAS and toluidine blue tests (Smyth \& Halton 1983).

The distribution of vesicles along the tegument was not uniform. The vesicles with fibrous contents were always present, but the spherical electron-dense forms were only seen in some areas and then in great numbers. The small, elongate electron-dense bodies were the most difficult to discern, not only because of their size, but their limited distribution within the tegument. They were always found close to the numerous thin projections of the basal membrane. Considering their form and location, it is possible that these electron-dense bodies exhibit an elongate shape only during the transition from the inner to external tegument while passing through the thin connection which may represent the cytoplasmic link between the cell bodies and the external syncytium. Subsequently, they would return to the oval or spherical shape found not only in the cell bodies but also in the syncytium. If this is true, then only two types of vesicles are present, i.e. those with fibrous contents and those that are electron-dense which differs from the three types reported by Justine (1992).

The chemical composition of the vesicles has been little studied. Lyons (1973) stated that the electron-dense granules may be composed of mucoproteins, since they are slightly alcian blue-PAS positive and diastase labile. Ramasamy and Bhuvaneswary (1993), when describing four types of vesicles in the tegument of Gotocotyla bivaginalis, also referred to similar patterns described by other authors which still needed additional study on their chemical composition. The alcian blue and E-PTA cytochemical tests were 
performed for the first time in monogeneans in this study in order to clarify the composition of these vesicles, but positive results were obtained for mucopolysacharides and basic proteins only in the electron-dense vesicles of $A$. heterodus. The vesicles with a fibrous content similar to the fibrous matrix of the parenchyma gave negative results with the tests used.

The location and great number of alcian blue positive vesicles found not only in the cell bodies and along the cytoplasmic extensions but also in the external tegument suggested a movement of these vesicles, although whether the process was endocytosis or exocytosis was not determined. Exocytosis in the tegument of monogeneans had been previously referred to by Ramasamy et al. (1986, 1987, 1995), who discussed the possibility of the discharge of material from these vesicles being part of the glycocalyx or of the mucous layer. Another possibility to explain the flux of these vesicles was discussed by Smyth and Halton (1983) when they suggested that the syncytium would have a vital role in the trans-tegumental nutrition and metabolism of the Monogenea. Other cytochemical tests are still necessary to clarify the function of vesicles found in $A$. heterodus .

\section{REFERENCES}

Benhnke O, Zelander T 1970. Preservation of intercellular substances by the cationic dye Alcian blue in preparative procedures for electron microscopy. $J$ Ultrastr Res 31: 424.

Bloom FE, Aghajanian GK 1968 Fine structure and cytochemical analysis of the staining of synaptic junctions with phosphotungstic acid. J Ultrastr Res 22: 361-375.

Bretscher A 1991. Microfilament structure and function in the cortical cytoskeleton. Ann Rev Cell Biol 7: 337-374.

Euzet L, Maillard C 1973. Sur deux microcotylidae (Monogenea) parasites branchiaux de téléostéen du genre Diplodus (Sparidae). Bull MNHN 3, serie Zoologie, 101: 795-805.

Justine J-L 1992. Ultrastructure of spermiogenesis, spermatozoa and the tegument of Atriaster sp. (Platy- helminthes, Monogenea, Polyopisthocotylea, Microcotylidae). Zool Scripta 21: 231-238.

Lyons KM 1973. The epidermis and sense organs of the monogenea and some related groups. Adv Parasitol 11: 193-232.

McMannus JFA, Mowry RW 1960. Staining Methods. Histhologic and Histhochemical, Paul B Hoeber, Inc. Medical Division of Harper \& Brothers, New York, $423 \mathrm{pp}$.

Mamaev Y L 1984. The composition of the genera Atriaster and Atrispinum (Microcotylidae, Monogenea) and some aspects of their morphology. Parazitologiya 18: 204-208.

Pearse AGE 1968. Histochemistry - Theoretical and Applied, Vol I, J \& A Churchill Ltd., London, 355 pp.

Ramasamy P, Bhuvaneswari R 1993. The ultrastructure of the tegument and clamp attachment organ of Gotocotyla bivaginalis (Monogenea, Polyopisthocotylea ). Int J Parasitol 23: 213-220.

Ramasamy P, Brennan GP, Halton DW 1995. Ultrastructure of the surface structures of Allodiscocotyla diacanthi (Polyopisthocotylea: Monogenea) from the gills of the marine teleost fish, Scomberoides tol. Int J Parasitol 25: 43-54.

Ramasamy P, Hanna R, Threadgold LT 1986. The surface topography and ultrastructure of the tegument and the haptor of Pricea multae (Monogenea). Int $\mathrm{J}$ Parasitol 16: 581-589.

Ramasamy P, Hanna R, Threadgold LT 1987. Scanning and transmission electron microscopic studies of the surface of Vallisia indica (Monogenea, Polyopisthocotylea). Int J Parasitol 17: 1187-1195.

Santos CP, Souto-Padron T, Lanfredi RM 1996. Atriaster heterodus Lebedev \& Parukhin, 1969 and Polylabris tubicirrus (Paperna \& Kohn, 1964) (Monogenea) from Diplodus argenteus (Val., 1830) (Teleostei: Sparidae) from Brazil. J Helm Soc Washington 63: 181-187.

Santos CP, Souto-Padrón T, Lanfredi RM 1998. The ultrastructure of the gastrodermis and the nutrition of the gill parasitic Atriaster heterodus Lebedev and Paruchin, 1969 (Platyhelminthes: Monogenea). Mem Inst Oswaldo Cruz 92: 277-282.

Smyth J, Halton DW 1983. The Physiology of Trematodes, 2nd ed., Cambridge University Press, Cambridge, $446 \mathrm{pp}$. 